\title{
Short communication \\ Association of serum adenosine deaminase level with atherosclerotic index in type 2 diabetes mellitus patients
}

\author{
Rasheed Khan M. ${ }^{1}$, Vinod Babu S. ${ }^{2}$, Kuzhandai Velu V. ${ }^{3}$ \\ ${ }^{1}$ Assistant Professor, Department of Biochemistry, Trichy SRM Medical College, Hospital and Research \\ Centre, Irungalur, Trichy, 621105, Tamil Nadu, India \\ ${ }^{2}$ Associate Professor, ${ }^{3}$ Assistant Professor, Department of Biochemistry, Mahatma Gandhi \\ Medical College and Research Institute, SBV, Pillaiyarkuppam, 607 402, Pondicherry, India
}

(Received: October $2020 \quad$ Revised: February $2021 \quad$ Accepted: February 2021)

Corresponding author: Vinod Babu S. Email: drvinodbabu@gmail.com

\begin{abstract}
Introduction and Aim: The atherosclerosis is the major cause of morbidity and mortality among diabetes population. Diabetes mellitus can accelerate atherosclerotic processes. Adenosine deaminase (ADA) plays a significant role in both glucose and lipid metabolism through adenosine. This study aimed to correlate the atherosclerotic index with adenosine deaminase levels in Type 2 diabetes mellitus patients. The aim of the study is to find the association between serum ADA levels with atherosclerotic index.
\end{abstract}

Materials and Methods: A cross sectional study conducted in 100 subjects (50 control and 50 T2DM patients). The following biochemical parameter were estimated: total cholesterol, triacylglycerol, HDL- C and ADA. VLDL, LDL and other atherosclerotic index were calculated using formulae. Statistical analysis such as Student' $s^{`} t$ ' test and Pearson's correlation were performed.

Results: We found significant increase ( $\mathrm{p}$ value $<0.001$ ) in lipid profile, Non-HDL-C and lipid ratio when compared to T2DM with control group. The correlation of serum ADA with lipid profile and lipid ratio did not show any correlation.

Conclusion: Serum ADA used as a biomarker for evaluation of glycemic status. ADA was insignificant, when correlated with dyslipidemia and atherosclerotic index.

Keywords: ADA; atherosclerotic index; T2DM; lipid profile.

\section{INTRODUCTION}

$\mathrm{D}$ iabetes is a disorders characterized by variable degrees of insulin resistance, impaired insulin secretion and increased glucose production (1). Prevalence of Type 2 Diabetes Mellitus (T2DM) is a global public health threat (2-4). The metabolic disturbance causes secondary pathophysiologic changes in multiple organ systems. This imposes a tremendous burden on the individual as well as health care system of both developed and developing countries.

Hyperlipidemia characterized

by hypertriglyceridemia, increased LDL, decreased HDL,

high apolipoprotein B and low apolipoprotein A-1 (5). Literature indicates that lipid accumulation also represents a chronic inflammatory process $(6,7)$.

Adenosine acts on glucose and lipid metabolism, will be similar to insulin action. By reduces free fatty acid levels and increases sensitivity of insulin. As ADA enzyme regulate bioactivity of insulin and decreasing glucose uptake by inhibiting adenosine (8-10). ADA increases lipolysis that leading to insulin resistance. We found there is association of adenosine with insulin and lipid metabolism this study was undertaken to evaluate the role of ADA in T2DM and to examine its correlation with lipid profile that will help us in early detection of cardiovascular complication.

\section{MATERIALS AND METHODS}

A cross-sectional study conducted for a period of one year from 2013- '14, in the Department of Internal Medicine and Department of Biochemistry at Narayana General Hospital, Nellore. 100 Subjects were selected with the age group between $35-70$ years, divided into two groups, 50 subjects diagnosed with T2DM and 50 healthy subjects served as control.

They were included based on the following criteria: Known diabetic patients for at least one-year duration with normal hepatic function. Subjects are excluded based on the following criteria: any chronic diseases and any associated complications with diabetes. Patients taking drugs for thyroid disorder or any corticosteroids, lipid-lowering drugs, oral contraceptives, aspirin, sulphonamides and pregnant women were also excluded.

Five $\mathrm{ml}$ blood sample was collected in fasting condition from each subject, and it was transferred into the grey tube for glucose analysis, lavender tube for HBA1c and plain red tube for ADA and Lipid Profile (TC, TG, HDL).

For statistical analysis data was collected and entered 
using Microsoft Excel ${ }^{\mathrm{TM}}$. The mean and standard deviation were calculated for all the Biochemical parameters. The significance between the groups was determined using Student t-test for equality of mean. The $p$ value of $<0.05$ was considered significant.

\section{RESULTS}

The mean age was approximately 51 year in both the groups. Out of 100 subjects, $43(86 \%)$ males and 7 (14\%) females were in T2DM group and 39 (78\%) and $11(22 \%)$ were in control group. There was a significant increase $(\mathrm{p}$ value $<0.001)$ in glycemic index (FBS, PPBS, and HbA1c), as shown in Table 1.

We found significant increase ( $p$ value $<0.001$ ) in lipid profile (TC, TGL, HDL, LDL, and VLDL), Non-HDL-C and lipid ratio (LDL/HDL, TC/TG, TG/HDL and Non-HDL/HDL) when compare T2DM with control group which has been depicted in Table 1. The correlation of serum ADA with lipid profile and lipid ratio (Table. 2) didn't show any correlation within the groups of T2DM and control groups.

Table 1: Base line and biochemistry characteristics of study and control group

\begin{tabular}{|c|c|c|c|}
\hline Variables & T2DM & Control & p Value \\
\hline Age (years) & $52.96 \pm 7.951$ & $50.34+8.494$ & 0.115 \\
\hline FBS (mg/dL) & $193.02 \pm 56.008$ & $90.52+11.682$ & $<.001^{\mathrm{a}}$ \\
\hline PPBS (mg/dL) & $243.5 \pm 40.991$ & $137.44+7.234$ & $<.001^{\mathrm{a}}$ \\
\hline HbA1c (\%) & $8.334 \pm 1.952$ & $4.764 \pm 0.406$ & $<.001^{\mathrm{a}}$ \\
\hline ADA (U/L) & $34.92 \pm 2.522$ & $14.1+2.613$ & $<.001^{\mathrm{a}}$ \\
\hline TC $(\mathrm{mg} / \mathrm{dL})$ & $223.94 \pm 39.991$ & $183.72+40.501$ & $<.001^{\mathrm{a}}$ \\
\hline TG $(\mathrm{mg} / \mathrm{dL})$ & $182.86 \pm 56.272$ & $115.88 \pm 36.976$ & $<.001^{\mathrm{a}}$ \\
\hline HDL $(\mathrm{mg} / \mathrm{dL})$ & $42.62 \pm 8.696$ & $51.86 \pm 10.359$ & $<.001^{\mathrm{a}}$ \\
\hline LDL $(\mathrm{mg} / \mathrm{dL})$ & $145.32 \pm 40.851$ & $108.5 \pm 32.222$ & $<.001^{\mathrm{a}}$ \\
\hline VLDL $(\mathrm{mg} / \mathrm{dL})$ & $36.572 \pm 11.254$ & $23.176 \pm 7.395$ & $<.001^{\mathrm{a}}$ \\
\hline Non-HDL (mg/dL) & $181.32 \pm 42.681$ & $131.86 \pm 34.675$ & $<.001^{\mathrm{a}}$ \\
\hline LDL/HDL & $3.637 \pm 1.597$ & $2.123 \pm 0.607$ & $<.001^{\mathrm{a}}$ \\
\hline TC/TGL & $1.319 \pm 0.392$ & $1.723 \pm 0.581$ & $<.001^{\mathrm{a}}$ \\
\hline TG/HDL & $4.449 \pm 1.467$ & $2.286 \pm 0.769$ & $<.001^{\mathrm{a}}$ \\
\hline Non-HDL/HDL & $4.512 \pm 1.737$ & $2.584 \pm 0.656$ & $<.001^{\mathrm{a}}$ \\
\hline
\end{tabular}

Note: Student's t-test. a Levene's test is significant $(\mathrm{p}<.05)$, suggesting a violation of the equal variance assumption

Table 2: Pearson's correlation between serum ADA vs Non-HDL and Lipid ratios

\begin{tabular}{|c|c|c|c|c|}
\hline \multirow{2}{*}{\begin{tabular}{c}
\multirow{2}{*}{$\begin{array}{c}\text { Correlation } \\
\end{array}$} \\
\cline { 2 - 5 }
\end{tabular}} & \multicolumn{3}{|c|}{ ADA } \\
\hline r value & P value & r value & P value \\
\hline LDL/HDL & -0.074 & 0.609 & 0.109 & 0.452 \\
\hline T Chol/TGL & -0.075 & 0.603 & -0.166 & 0.251 \\
\hline TGL/HDL & 0.027 & 0.852 & 0.241 & 0.092 \\
\hline Non-HDL/HDL & -0.063 & 0.662 & 0.172 & 0.233 \\
\hline Non-HDL & -0.056 & 0.701 & 0.172 & 0.233 \\
\hline \multicolumn{2}{|c|}{$* \mathrm{p}<.05, * * \mathrm{p}<.01, * * * \mathrm{p}<.001$} \\
\hline
\end{tabular}

\section{DISCUSSION}

Diabetes mellitus is a common metabolic disorder of the endocrine system and is a leading cause of death globally. It is characterized by increased levels of plasma blood glucose due to multiple factors that may be hereditary, environmental impact mainly diet or associated insulin resistance $(11,12)$. It may lead to several complications in due course of time which could lead to disability and morbidity in patients (13). The incidence of patients with type 2 diabetes mellitus has been increasing rapidly in the past 2-3 decades and there is notably higher prevalence of associated with cardiometabolic risk factors (14).
Present study found significant increased serum Adenosine deaminase (ADA) activity in T2DM subjects when compared with control group, as shown in Table.1. Hoshino et al., also documented increased levels of ADA activity and don't show any significant correlation with lipid profile (15). Increased ADA activity inhibits the action of adenosine which mimic the action of insulin in the adipose tissue and skeletal muscles through GLUT-4 receptors. Depletion of adenosine leads to insulin resistant, subsequent cause's hyperglycemia. $(15,16)$ Lee et al., and Shiva Prakash et al., reported that ADA activity is comparatively reduced in T2DM patients who are under good glycemic control (13, 
16).

Inhibition of adenosine increases CAMP, this directed to increase in circulation of free fatty acids. These FFA are converted into triglycerides and get deposited in the adipose tissue (17). This excess storage of triglyceride stimulated pro-inflammatory responses such as interleukins and another factor. Based on the above mechanism, we tried to find association of ADA activity with lipid profile and atherosclerotic index. We found significant increase ( $\mathrm{p}$ value $<0.001$ ) in lipid profile (TC, TGL, HDL, LDL, and VLDL), Non-HDL-C and lipid ratio (LDL/HDL, TC/TG, TG/HDL and Non-HDL/HDL) when compare T2DM with control group which has been depicted in Table 1. The correlation of serum ADA with lipid profile and lipid ratio (Table 2) did not show any correlation within the groups of T2DM and control groups.

ADA alongside other immune modulating enzymes acts as a deconstructive oxidative marker in diabetes and plays an important role in progression in its complications.(16) The indication of increase in ADA may serve as a glycemic marker in of T2DM. Role of ADA activity along with lipid profile and atherosclerotic index score was not statistically significant.

\section{CONCLUSION}

Assessment of serum ADA level is found to be a cost-effective biomarker and its efficient use may help in assessing cell-mediated immunity in diabetic individuals. Thus, we conclude that increased ADA activity may be an indicator in the immunopathogenesis of T2DM and can also be implicated as a biomarker for predicting glycemic control individuals with T2DM, but there were no significant findings noted in the assessment of dyslipidemia and atherosclerotic index. Further studies are required, especially the elucidation of the role of ADA levels on the lipid profile of T2DM patients with fair to poor control.

\section{CONFLICT OF INTEREST}

Authors declare no conflicts of interest.

\section{REFERENCES}

1. Diagnosis and Classification of Diabetes Mellitus. Diabetes Care. 2009 Jan; 32(Suppl 1): S62-S67.

2. Ramachandran, A., Snehalatha, C., Kapur, A., Vijay, V., Mohan, V., Das, A. K., et al., High prevalence of diabetes and impaired glucose tolerance in India: National Urban Diabetes Survey. Diabetologia. 2001 Sep; 44(9): 1094-1101.

3. Shrivastava, S. R., Ghorpade, A. G. High prevalence of type 2 diabetes melitus and its risk factors among the rural population of Pondicherry, South India. J Res Health Sci. 2014 Jan 11; 14(4): 258-263.

4. Kumar, P., Mallik, D., Mukhopadhyay, D. K., Sinhababu, A., Mahapatra, B. S., Chakrabarti, P. Prevalence of diabetes mellitus, impaired fasting glucose, impaired glucose tolerance, and its correlates among police personnel in Bankura district of west Bengal. Indian J Public Health. 2013 Jan 1; 57(1): 24.
5. Martín-Timón I. Type 2 diabetes and cardiovascular disease: Have all risk factors the same strength? World J Diabetes. 2014; 5(4): 444.

6. Ridker, P. M. Clinical application of C-reactive protein for cardiovascular disease detection and prevention. Circulation. 2003 Jan 28; 107(3): 363-369.

7. Fu, Y., Wu, Y., Liu, E. C-reactive protein and cardiovascular disease: From animal studies to the clinic (Review). Exp Ther Med. 2020 Jun 4; 20(2): 1211-1219.

8. Sapkota, L. B., Thapa S, Subedi N. Correlation study of adenosine deaminase and its isoenzymes in type 2 diabetes mellitus. BMJ Open Diabetes Res Care. 2017 Mar; 5(1): e000357.

9. Hariprasath, G., Ananthi, N. Glycemic control and raised adenosine deaminase activity in type 2 diabetes mellitus. IOSR J Dent Med Sci. 2017 Jan; 16(01): 53-56.

10. Patel, B., Taviad, D., Malapati, B., Chhatriwala, M., Shah, R. Serum adenosine deaminase in patients with type-2 diabetes mellitus and its relation with blood glucose and glycated haemoglobin levels. Int J Biomed Res. 2014 Sep 30; 5(9): 556.

11. Singh, P., Khan, S., Rabindra Kumar, M. Adenosine deaminase activity and its relation with glycated hemoglobin and uric acid in type 2 diabetic patients. IJDO. 2013 Jan 1; 5(1): 1-6.

12. Niraula, A., Thapa, S., Kunwar, S., Lamsal, M., Baral, N., Maskey, R. Adenosine deaminase activity in type 2 diabetes mellitus: does it have any role? BMC Endocr Disord. 2018 Aug 20; 18(1): 58.

13. Lee, J-G., Kang, D. G., Yu, J. R., Kim, Y. R., Kim, J. S., Koh, G. P., et al. Changes in adenosine deaminase activity in patients with type 2 diabetes mellitus and effect of dpp-4 inhibitor treatment on ada activity. Diabetes Metab J. 2011; 35(2): 149.

14. Wu, C-J., Yu, Z-R. Effects on blood glucose, insulin, lipid and proatherosclerotic parameters in stable type 2 diabetic subjects during an oral fat challenge. Lipids Health Dis. 2004; 3(1): 17.

15. Hoshino, T., Yamada, K., Masuoka, K., Tsuboi, I., Itoh, K., Nonaka, K., et al. Elevated adenosine deaminase activity in the serum of patients with diabetes mellitus. Diabetes Res Clin Pract. 1994 Sep; 25(2): 97-102.

16. Larijani, B., Heshmat, R., Ebrahimi-Rad, M., Khatami, S., Valadbeigi, S., Saghiri, R. Diagnostic value of adenosine deaminase and its isoforms in type 2 diabetes mellitus. Enzyme Res. 2016; 2016: 1-6.

17. Mills, S. E. Regulation of porcine adipocyte metabolism by insulin and adenosine. J Anim Sci. 1999; 77(12): 3201. 